stents for treating intracranial aneurysms. Intervent Neurol 2019;8:38-54

15 Turk AS III, Martin RH, Fiorella D, Mocco J, Siddiqui A, Bonafe A. Flow diversion versus traditional endovascular coiling therapy: design of the prospective LARGE aneurysm randomized trial. Am J Neuroradiol 2014;35(7):1341-1345

16 Hammoud D, Gailloud P, Olivi A, Murphy KJ. Acute vasogenic edema induced by thrombosis of a giant intracranial aneurys a cause of pseudostroke after therapeutic occlusion of the parent vessel. Am J Neuroradiol 2003;24(6):1237-1239

17 Berge J, Tourdias T, Moreau J-F, Barreau X, Dousset V. Perianeurysmal brain inflammation after flow-diversion treatment. Am J Neuroradiol 2011;32(10):1930-1934
18 Saatci I, Yavuz K, Ozer C, Geyik S, Cekirge HS. Treatment of intracranial aneurysms using the pipeline flow-diverter embolization device: a single-center experience with long-term follow-up results. Am J Neuroradiol 2012;33(8):1436-1446

19 Raymond J, Gentric JC, Darsaut TE, et al. Flow diversion in the treatment of aneurysms: a randomized care trial and registry. J Neurosurg 2017;127(3):454-462

20 Rouchaud A, Brinjikji W, Lanzino G, Cloft HJ, Kadirvel R, Kallmes DF. Delayed hemorrhagic complications after flow diversion for intracranial aneurysms: a literature overview. Neuroradiology 2016;58(2):171-177

\title{
Anesthetic Management of a Patient with Pacemaker Undergoing Clipping of Intracranial Aneurysm
}

Summit D. Bloria ${ }^{1}$ Shiv Lal Soni ${ }^{1} \quad$ Ketan Kataria ${ }^{1} \quad$ Pallavi Bloria ${ }^{2}$

\author{
Address for correspondence Summit D. Bloria, MD, Department of \\ Anesthesia, Postgraduate Institute of Medical Education and Research, \\ c/o 3245 Sector 15D, Chandigarh, 160015, Punjab, India \\ (e-mail: summitbloria13@gmail.com).
}
Abstract
Keywords
- aneurysm
- cerebral aneurysm clipping
- pacemaker

Pacemaker devices have revolutionized the lives of patients with cardiac arrhythmias and have become increasingly common. Anesthetic management of patients with cardiac pacemakers provides special challenges. We describe our experience of a patient having implanted permanent pacemaker posted for clipping of ruptured intracranial aneurysm.

\section{Introduction}

Anesthetic management of patients with implanted pacemaker requires a thorough knowledge of the indication for pacemaker placement, present mode of pacemaker, and extent of dependency of the patient on pacemaker. In patients with pacemakers who are undergoing neurosurgery, the additional considerations include the proximity of operative site to the pacemaker, prolonged duration of surgery, potential for major amount of blood loss, cardiac insults
Published online

April 30, 2020
Dol https://doi.org/

$10.1055 / \mathrm{s}-0039-1685248$

ISSN 2348-0548.
(C2020. Indian Society of Neuroanaesthesiology and Critical Care. This is an open access article published by Thieme under the terms of the Creative Commons Attribution-NonDerivative-NonCommercial-License, permitting copying and reproduction so long as the original work is given appropriate credit. Contents may not be used for commercial purposes, or adapted, remixed, transformed or built upon. (https://creativecommons.org/licenses/by-nc-nd/4.0/).

Thieme Medical and Scientific Publishers Pvt. Ltd. A-12, 2nd Floor, Sector 2, Noida-201301 UP, India 




Fig. 1 Chest X-ray of the patient showing pacemaker in left pectoral area.

caused by neurological causes (aneurysmal subarachnoid hemorrhage [aSAH], traumatic brain injury), etc. aSAH affects the cardiac status immensely. Patients with aSAH patients can present with new-onset electrocardiographic (ECG) changes, new-onset wall motion abnormalities, and elevation in cardiac troponin levels. ECG changes are seen in around 40 to $100 \%$ of patients with SAH and include sinus bradycardia, sinus tachycardia, atrioventricular dissociation, and ventricular arrhythmias. ${ }^{1,2}$ The stimulation caused by increased amounts of catecholamines is considered to be the cause of these cardiac manifestations. These effects can be magnified in patients with implanted pacemaker.

\section{Case Report}

A 62-year-old male patient, a case of symptomatic complete heart block who had undergone permanent pacemaker insertion for complete heart block (presently on dual-chamber rate adaptive pacemaker [DDDR] mode) around 6 months back ( - Fig. 1 ), suffered from sudden onset of severe headache one morning associated with one episode of projectile vomiting and giddiness. The patient was taken to a nearby hospital, and noncontrast computed tomography (NCCT) of the head was performed, which revealed bilateral sylvian fissure SAH. The patient was then referred to our hospital where a CT angiography was performed, which demonstrated a ruptured left distal anterior cerebral artery aneurysm (-Fig. 2). After discussion with the patient and his attendants, a decision to perform an emergency craniotomy and aneurysmal clipping was taken.

On examination during preanesthetic checkup, the patient was conscious, cooperative, and oriented, and his vitals were

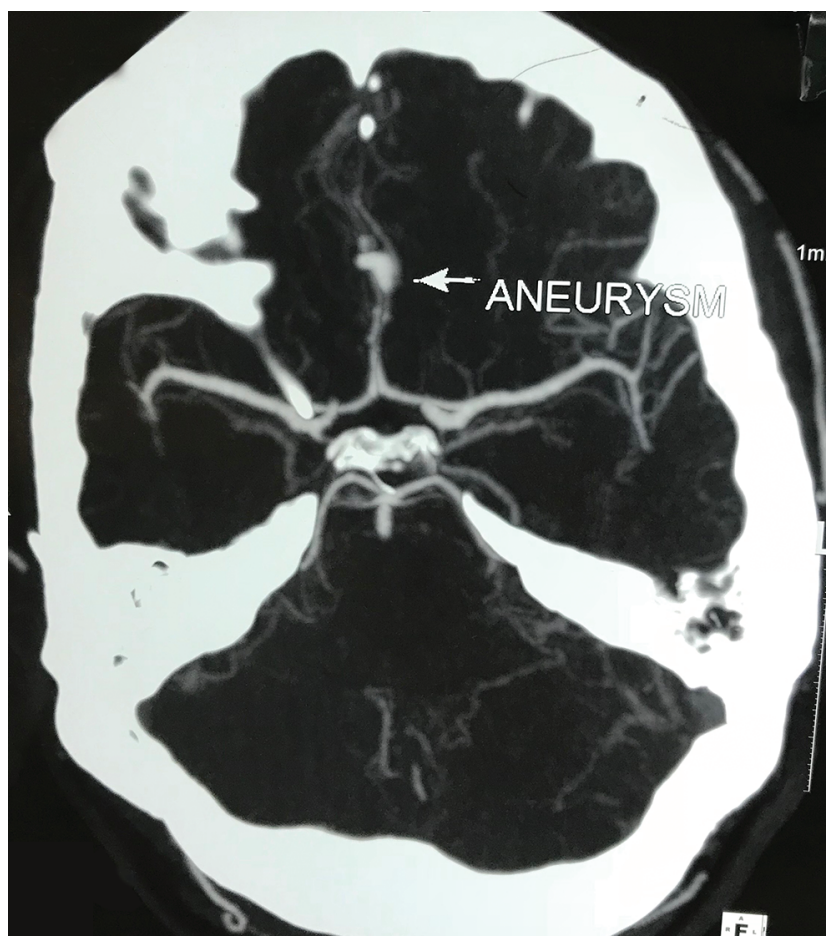

Fig. 2 CT angiography demonstrating DACA aneurysm. CT, computed tomography. DACA, distal anterior cerebral artery.

within the normal limits. His preoperative Glasgow coma scale score was E4V5M6. He was classified as Hess and Hunt grade 2, World Federation of Neurosurgeon (WFNS) grade 1, and modified Fischer's grade 4. Airway evaluation was unremarkable. Blood biochemistry was within normal limits. Chest X-ray showed intact leads of pacemaker, and ECG showed sinus rhythm with paced beats. Echocardiography showed good left ventricular systolic function with ejection fraction of 50\%. The cardiologist's opinion was taken, who advised changing the pacemaker to asynchronous mode prior to surgery with a heart rate of 75 beats/min. The pacemaker was evaluated, and it was found to be working fine.

The pacemaker was subsequently shifted to asynchronous mode with a heart rate of 75 beats/min on the day of surgery by the pacemaker company representative. The need to avoid use of monopolar cautery and minimize the use of bipolar cautery was discussed with the surgeon.

On the day of surgery, the patient was premedicated with tab ranitidine $150 \mathrm{mg}$, and $1 \mathrm{mg}$ IV midazolam was given to allay anxiety in preoperative room under monitoring. A 20G arterial cannula was placed in the left radial artery under local anesthesia and baseline arterial blood gas (ABG) analysis was done. After shifting the patient to operating theater, standard monitors including NMT (neuromuscular transmission) monitors were attached. Injection isoproterenol, atropine, and external transcutaneous defibrillator and pacemaker were kept ready. Cardiology backup was taken. Induction was done with fentanyl $200 \mu \mathrm{g}$, propofol $130 \mathrm{mg}$, and vecuronium $10 \mathrm{mg}$. Endotracheal intubation was done with endotracheal tube of internal diameter of $8.5 \mathrm{~mm}$ after the train of four count 
decreased to zero. A right subclavian central venous line was inserted after induction under ultrasound guidance. Anesthesia was maintained with propofol infusion, vecuronium top-ups in $67 \%$ nitrous oxide: $33 \%$ oxygen mixture. Intraoperative volume status was monitored with central venous pressure (CVP), systolic pressure variation (SPV), and a pulse pressure variation (PPV) value. Intraoperative SPV values were kept below $10 \mathrm{~mm} \mathrm{Hg}$. Surgery lasted for 4 hours. The cardiac status of the patient remained stable throughout the duration of surgery, with no abnormal rhythm or hemodynamic instability. Intraoperative blood loss was 1,500 mL, and packed red blood cell (PRBC) transfusion was started when the blood loss exceeded the maximal allowable blood loss of the patient. The pacemaker was evaluated postoperatively and was shifted to DDDR mode. Postoperatively, the patient had to be tracheostomized in view of failure to wean from ventilator. He was discharged to home with E2VTM3 status.

\section{Discussion}

The perioperative period for patients with pacemakers poses unique challenges. Effective communication with the physician caring for the pacemaker is imperative for patient's safety. The most common problem intraoperatively is electromagnetic interference due to cautery devices. These devices produce a high energetic electromagnetic field at a frequency that passes through the filters of pacemakers leading to oversensing. This oversensing leads to a pacemaker inhibition and may cause cardiac arrest. Therefore, for surgical procedures using electrocautery devices, pacemaker-dependent patients should be programmed into an asynchronous pacing mode. ${ }^{3}$

Also, aSAH causes multitude of effects on the heart, including arrythmias. ${ }^{4}$ It has been documented that 40 to $100 \%$ of patients with SAH have new-onset ECG abnormalities, including even ventricular tachycardia and fibrillation; new-onset wall motion changes and depressed left ventricular function are seen in around 13 to $18 \%$ of patients. Hence, our patient's cardiac status had suffered a dual insult: due to the conduction disorder that necessitated pacemaker insertion and due to aSAH. Hence, we decided to have a preoperative evaluation of the pacemaker echo in our patient although the present recommendations do not make it compulsory. Another important consideration was whether to insert a subclavian line in this patient or not. Craniotomies can result in excessive blood loss, and need for inotropes may arise. However, during insertion of a central venous line in a patient with a pacemaker, there is a theoretical possibility of pacemaker getting displaced. Also, pacemakers can cause venous complications, making central venous line placement difficult. ${ }^{5}$

Use of nitrous oxide in patients with recently inserted pacemakers has been documented to cause pacemaker dysfunction due to formation of air pockets. ${ }^{6}$

When the patient is on asynchronous mode, occurrence of hypovolemia will not be accompanied with reflex tachycardia, and this must be kept in mind while caring for the patient intraoperatively. Hence, we used dynamic measures of fluid responsiveness such as SPV and PPV in the management of this patient along with measurement of CVP. We could also have used other dynamic predictors of fluid volume status and fluid responsiveness indicators such as FloTrac and inferior vena cava (IVC) distensibility index, to guide intravenous fluid therapy in our patient. However, monetary considerations and limited access to the patient intraoperatively prevented us from employing them to guide fluid administration intraoperatively.

\section{Conflict of Interest}

None declared.

\section{References}

1 Lanzino G, Kongable GL, Kassell NF. Electrocardiographic abnormalities after nontraumatic subarachnoid hemorrhage. J Neurosurg Anesthesiol 1994;6(3):156-162

2 Jangra K, Grover VK, Bhagat $\mathrm{H}$, et al. Evaluation of the effect of aneurysmal clipping on electrocardiography and echocardiographic changes in patients with subarachnoid hemorrhage: a prospective observational study. J Neurosurg Anesthesiol 2017;29(3):335-340

3 Niehaus M, Tebbenjohanns J. Electromagnetic interference in patients with implanted pacemakers or cardioverterdefibrillators. Heart 2001;86(3):246-248

4 Ahmadian A, Mizzi A, Banasiak M, et al. Cardiac manifestations of subarachnoid hemorrhage. Heart Lung Vessel 2013;5(3):168-178

5 Spittell PC, Hayes DL. Venous complications after insertion of a transvenous pacemaker. Mayo Clin Proc 1992;67(3):258-265

6 Lasala AF, Fieldman A, Diana DJ, Humphrey CB. Gas pocket causing pacemaker malfunction. Pacing Clin Electrophysiol 1979;2(2):183-185 